\title{
Developmental expression of the cucumber Cs-XTH1 and Cs-XTH3 genes, encoding xyloglucan endotransglucosylase/hydrolases, can be influenced by mechanical stimuli
}

\author{
Robert Malinowski ${ }^{1,5} \cdot$ Stephen C. Fry ${ }^{2} \cdot$ Sabina Zuzga ${ }^{1}$. Anita Wiśniewska ${ }^{3}$ Michał Godlewski ${ }^{4}$. \\ Andrzej Noyszewski ${ }^{1,6} \cdot$ Anna Barczak-Brzyżek ${ }^{1} \cdot$ Stefan Malepszy $^{1} \cdot$ Marcin Filipecki $^{1}$ (1)
}

Received: 27 September 2017 / Revised: 14 February 2018 / Accepted: 19 June 2018 / Published online: 29 June 2018

(c) The Author(s) 2018

\begin{abstract}
The expression of two genes encoding xyloglucan endotransglucosylase/hydrolases (XTHs), Cs-XTH1 and Cs-XTH3, was upregulated during the onset of cucumber somatic embryogenesis. As a means of characterising the developmental regulation of these genes, the activity of the respective upstream regulatory regions was investigated in seedlings and somatic embryos of Arabidopsis thaliana and Cucumis sativus. GUS assays revealed that both genes are under developmental control. In addition, elevated promoter activity was found in the tension-bearing regions of the plant and in response to touch and wounding, which is consistent with the existence of numerous stress-related cis elements in the 5 '-regulatory regions. In vivo xyloglucan endotransglucosylase (XET) action assays were performed to gain an overview on the role of XTHs during somatic embryogenesis. The highest XET action was observed in the external cell layers of somatic embryos in the cotyledonary region and in the presumptive region of peg formation. Based on the results, we propose a dual mechanism (one developmental and the second adaptive) for the regulation of $\mathrm{Cs}$-XTH1 and Cs-XTH3 activity wherein the developmental pattern can be modified by mechanical stimuli.
\end{abstract}

Keywords Xyloglucan endotransglucosylase/hydrolase (XTH) - Somatic embryogenesis · Cucumber · Cucumis sativus . Developmental and adaptive gene regulation · Xyloglucan-oligosaccharide-sulphorhodamine conjugate (XGO-SR)

Communicated by Manoj Prasad.

Electronic supplementary material The online version of this article (https://doi.org/10.1007/s11738-018-2707-7) contains supplementary material, which is available to authorized users.

Marcin Filipecki

marcin_filipecki@sggw.pl

1 Department of Plant Genetics, Breeding and Biotechnology, Faculty of Horticulture, Biotechnology and Landscape Architecture, Warsaw University of Life Sciences, Nowoursynowska 159, 02-776 Warsaw, Poland

2 The Edinburgh Cell Wall Group, Institute of Molecular Plant Sciences, School of Biological Sciences, The University of Edinburgh, Daniel Rutherford Building, The King's Buildings, Mayfield Road, Edinburgh EH9 3BF, UK

3 Department of Plant Physiology, Faculty of Agriculture and Biology, Warsaw University of Life Sciences, Nowoursynowska 159, 02-776 Warsaw, Poland

\section{Introduction}

The XTHs are a family of plant genes encoding the xyloglucan transglucosylase/hydrolase enzymes (XTH; EC 2.4.1.207). These enzymes are capable of modulating the chemistry of the primary cell wall of plants, which consists

4 Department of Physiological Sciences, Faculty of Veterinary Medicine, Warsaw University of Life Sciences, Nowoursynowska 159, 02-776 Warsaw, Poland

5 Present Address: Department of Integrative Plant Biology, Institute of Plant Genetics PAS, Strzeszyńska 34, 60-479 Poznań, Poland

6 Present Address: Department of Horticultural Science, University of Minnesota, 305 Alderman Hall, 1970 Folwell Ave., St. Paul, MN 55108, USA 
of cellulose microfibrils embedded in a matrix of hemicellulosic and pectic polysaccharides, by remodelling the hemicellulose xyloglucan, the major component of the matrix in dicotyledonous plants. XTHs exhibit one or both of two enzymatic activities: the catalysis of the cleavage of a xyloglucan chain and subsequent religation to a different acceptor chain (xyloglucan endotransglucosylase activity-XET) and the catalysis of the cleavage of a xyloglucan chain with religation to water as an acceptor molecule (xyloglucan endohydrolase activity-XEH) (Eklöf and Brumer 2010; Franková and Fry 2013).

The enzymatic XET and XEH activities have been shown to lead to enhanced viscoelasticity or increased strength when applied to cellulose/xyloglucan biocomposite material mimicking a cell wall structure (Chanliaud et al. 2004), and exogenously applied XTHs have been shown to modify the extensibility of real plant cell wall material (Maris et al. 2009). These results have provided strong support for previous demonstrations of the ability of these proteins to integrate new xyloglucan molecules (Thompson et al. 1997) and to remodel existing xyloglucan molecules (Thompson and Fry 2001) of the cell wall. Based on these results, it would appear that by changing the mechanical properties of the cell wall, XTHs significantly influence the structural phenotype of the cell, thereby contributing to the regulation of the resulting plant phenotype. XTH enzymes are believed to be capable of strongly influencing the arrangement of cellular and tissue tensions in the plant. This type of morphological change, especially when it occurs locally, may be important for plant morphogenesis (Hejnowicz et al. 1977; Dumais et al. 2004).

The morphogenetic potential of XTH proteins should also be discussed in the light of the extensive number of genes that make up this family and the possible functional redundancy that may result from this. There are 33 XTH genes in the Arabidopsis genome, most of which exhibit individual tissue-specific and growth stage-dependent expression profiles (Yokoyama and Nishitani 2001). Similar family characteristics have been reported for other plants such as rice (29 members; Yokoyama et al. 2004), Populus trichocarpa (37 members; Ye et al. 2012) and the moss Physcomitrella patens (32 members; Yokoyama et al. 2010). Assuming that each cell type possesses its own specific "cell wall type", the diversity of expression patterns of XTH genes is consistent with the hypothesis that each "cell wall type" is characterised by a specific set of cell wall-modifying enzymes (Nishitani 2002).

There are several reports in the literature suggesting specific roles of XTH proteins in the regulation of intercellular relationships during cell differentiation. Antosiewicz et al. (1997) determined that the XTH22 protein is involved in intercellular space formation during xylem and phloem development in Arabidopsis. The transcription of the VaXTH1 and VaXTH2 genes during phloem formation in the azuki bean also supports the involvement of XTHs in the regulation of cell mechanical properties during plant development (Nakamura et al. 2003). Bourquin et al. (2002) observed that an XTH protein is involved in secondary cell wall formation in poplar (Populus tremula var. tremuloides) vascular tissue. Vissenberg et al. (2001) observed XET action [i.e. co-localisation of XET-active XTH(s) with the accessible endogenous donor substrate xyloglucan] in Arabidopsis roots at the site of root hair differentiation. Similar engagement of XTH proteins during root hair differentiation was shown in numerous evolutionarily distinct plants (Vissenberg et al. 2003). Similarly, testing promoter activities of root-specific Arabidopsis XTH genes confirmed previously observed expression profiles and the correlation of XET actions with different localizations and $\mathrm{pH}$ optima (Vissenberg et al. 2001, 2005).

Plant development is strongly modified by environmental factors. External stimuli often trigger the plant's adaptive response by regulating the mechanisms requiring specific growth modifications, of which cell wall remodelling is of great importance. XET enzyme activity seems to be no exception to this and appears to be an important element of the plant's response to mechanical stimuli or other stress factors (Potters et al. 2007, 2009). This phenomenon was first described for the TCH4 gene (XTH22) of Arabidopsis thaliana (Braam and Davis 1990) which was activated by environmental factors such as wind, rain or touch. The XTH22 protein accumulated in cells exhibiting adaptive changes to mechanical stress-i.e. in vascular and epidermis tissues (Antosiewicz et al. 1997). Moreover XTH22 gene transcription was activated in the dark or in response to low $\left(0{ }^{\circ} \mathrm{C}\right)$ or high $\left(35^{\circ} \mathrm{C}\right)$ temperatures (Braam and Davis 1990 ; $\mathrm{Xu}$ et al. 1995; Iliev et al. 2002). Xu et al. (1996) suggested that depending on the adaptive needs of the plant the XTH22 protein can cause local cell wall remodelling, leading to either temporary loosening or rapid reinforcement of the cell wall. However, extrapolations from gene transcription (or protein accumulation) to protein action can be misleading; for example, various factors such as an incompletely characterised heat-stable plant polymer (Sharples et al. 2017) can influence the activity of XTH proteins. A full understanding of the role of the XTH proteins in these processes requires assays for their activity and/or action (Vissenberg et al. 2000).

Linkiewicz et al. (2004), Malinowski et al. (2004) and Wiśniewska et al. (2012) reported an increase in the activity of two cucumber (Cucumis sativus L.) XTH genes, Cs$\mathrm{XTH} 1$ and $\mathrm{Cs}-\mathrm{XTH} 3$, during somatic embryo development. The localisation of their mRNAs in presumptive cotyledon regions suggested that these genes may play a role in embryo pattern formation. In continuation of this research, we report 
here our investigation of the mechanisms regulating the expression of these genes and XET action.

\section{Materials and methods}

\section{Plant material}

Cucumber (C. sativus L.) cv. Borszczagowski plants used for the analysis of promoter activity and for the induction of an embryogenic suspension culture were grown for 20-30 days under a $17 / 7$-h (light, $25^{\circ} \mathrm{C} /$ dark, $21^{\circ} \mathrm{C}$ ) photoperiod regime. The cucumber cell suspension culture was established and the induction of somatic embryogenesis was carried out according to the cytokinin model established by Burza and Malepszy (1998).

A. thaliana ecotype 'Columbia' plants were grown under a 10/14-h (light, $2{ }^{\circ} \mathrm{C} /$ dark, $19^{\circ} \mathrm{C}$ ) photoperiod regime. Flowering was induced by switching to a 14-h light period.

\section{Promoter::GUS (ß-glucuronidase) fusion constructs and plant transformation}

The PLACE (Higo et al. 1999) and PlantProm DB (Shahmuradov et al. 2003) databases were used to predict the cis elements. Predicted elements were arranged into developmentally- and stress-related groups according to database description for particular element (Fig. 1; for a detailed list of the cis elements predicted, see Table 1 of the supplementary data). Based on the distribution of predicted regulatory regions in the 5'-upstream sequence, we generated three promoter::GUS fusions each for $\mathrm{Cs}-\mathrm{XTHI}$ and $\mathrm{Cs}$-XTH3 (Fig. 1) in the binary pCAMBIA $1381 \mathrm{Z}$ vector (Hajdukiewicz et al. 1994). The main criterion applied to the construct design was the frequency of putative cis elements observed in particular region of the promoter analysed.

All constructs were used for the Arabidopsis transformation according to the Clough and Bent (1998) floral dip method. Transgenic plant selection was performed on GRODAN (GRODANIA A/S Doense, Hobro, Denmark) according to Hadi et al. (2002) with minor modifications. In brief, approximately 1000 seeds were sown on $15 \times 10 \times 2$-cm 'rockwool mate' watered with $1 / 4$-strength MS medium (Murashige and Skoog 1962) containing $5 \mathrm{mg}$ $1^{-1}$ hygromycin B (Duchefa, Haarlem, the Netherlands). Following matriconditioning at $4{ }^{\circ} \mathrm{C}$ for 3 days, the seeds were replaced in the growth chamber under vegetative growth conditions (see Materials and methods, plant material). After 3 weeks, resistant seedlings that had developed leaves were transferred to soil. For construct $-928 \mathrm{pCs}-$ XTH1, we obtained 8 independent transformation events, $-467 \mathrm{pCs}-\mathrm{XTH} 1-8,-275 \mathrm{pCs}-\mathrm{XTH} 1-26,-610 \mathrm{pCs}-$ XTH3-6, -430pCs-XTH3-2 and -244pCs-XTH3-43.

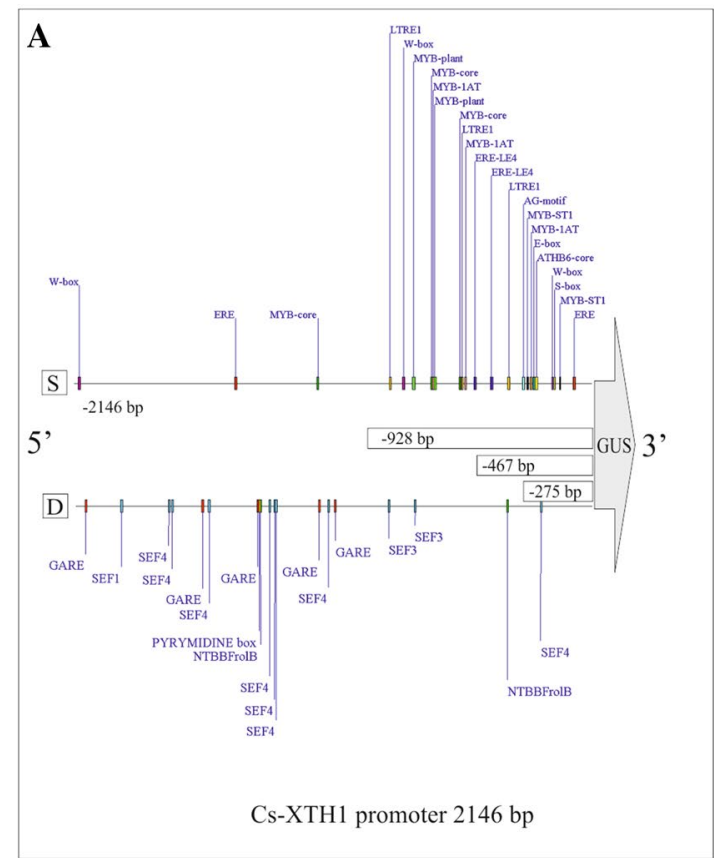

Fig. 1 Schematic representation of generated promoter::GUS fusions and the localisation of predicted cis elements in $5^{\prime}$ upstream regions of $C s-X T H 1$ (a) and $C s-X T H 3$ (b). The cis elements shown on the upper part of each scheme (S) represent stress-related elements; those

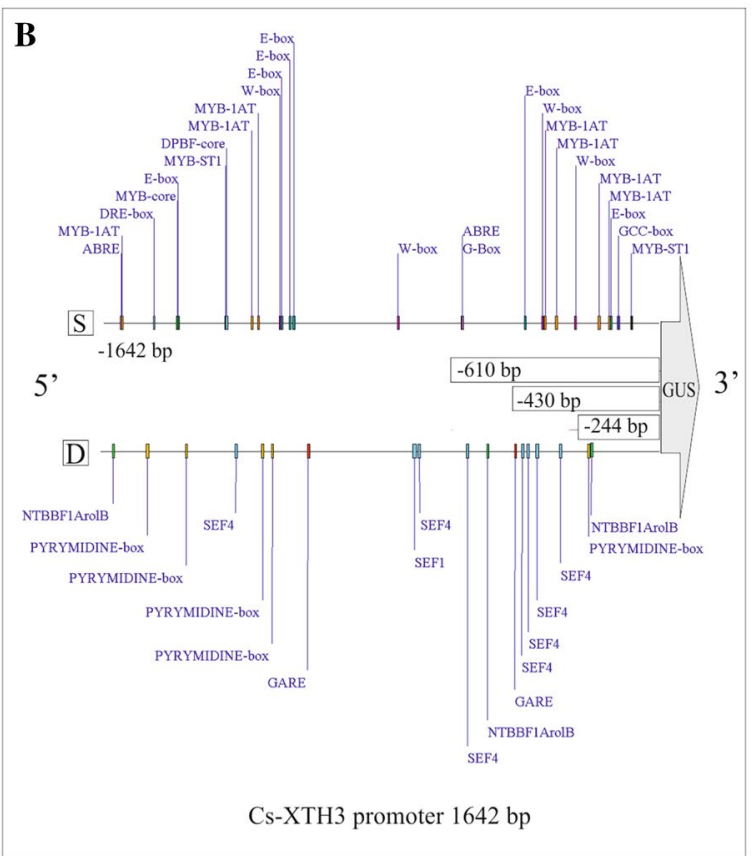

on the lower part are developmentally-related elements (d). For more detailed information on the localisation of the cis elements localization, see SupplementaryTable2 
Two constructs $(-928 \mathrm{pCs}-\mathrm{XTH} 1$ and $-610 \mathrm{pCs}-\mathrm{XTH} 3$; Fig. 1) were introduced into the cucumber genome. The Agrobacterium-mediated transformation of the cucumber embryogenic suspension culture was performed according to the method of Burza et al. (2006). For construct $-928 \mathrm{pCs}-\mathrm{XTH1}$, we obtained eight independent hygromycin-resistant embryogenic cell lines and regenerated five transgenic plants (independent transformation events), whereas transformation with $-610 \mathrm{pCs}-\mathrm{XTH} 3$ gave six embryogenic cell lines and no transgenic plants were regenerated. PCR analysis verified the presence of the transgene in T0 Arabidopsis and cucumber plants.

\section{GUS assay}

GUS activity was determined basically according to the method of De Block and Debrouwer (1992). Subsequent modifications to this method were designed on the basis of the experimental configuration.

To check the influence of wounding on the activity of the $\mathrm{Cs}-\mathrm{XTH} \mathrm{l}$ and $\mathrm{Cs}-\mathrm{XTH} 3$ promoters, we cut leaves three times with scissors at 30-min intervals ( $1 \mathrm{~h}$ between the first and last incision). Immediately after the last incision, the leaves were excised and placed in $1 \mathrm{mg} \mathrm{ml}^{-1} \mathrm{X}$-Gluc (Duchefa) working solution overnight (approximately 10-12 h). Wildtype cucumber and Arabidopsis leaves were used as controls. In an additional analysis, GUS activity in the leaves of old (2-3 weeks) and young (4-7 days) cucumber plants was compared. The results shown are representative for most of transformation events for each construct.

The assay for GUS activity in Arabidopsis seedlings was performed under conditions that eliminated any touch induction. The seeds of Arabidopsis $\mathrm{T}_{2}$ transgenic plants were sown on solid MS medium (2\% agar; Difco, Detroit Mich.) in vertically placed Petri dishes, and plants were grown at $22{ }^{\circ} \mathrm{C}$ for 7 days under light conditions (intensity: $150 \mu \mathrm{mol}$ $\left.\mathrm{m}^{-2} \mathrm{~s}^{-1}\right)$. GUS activity was assayed following tissue fixation in $2 \%(\mathrm{w} / \mathrm{v})$ paraformaldehyde by the same method as adopted for the XTH22 gene (Iliev et al. 2002).

Promoter activity was assayed in the cucumber somatic embryos by replacing the culture medium with the X-Gluc working solution (the same concentration as used above) followed by a 6-h incubation. The reaction was stopped with $70 \%$ ethanol, and the embryos and cell aggregates were observed under a stereomicroscope (Olympus SZ, Olympus Optical, Japan) or inverted microscope (Olympus BX60, Olympus Optical). For the whole-mount microscopic observation, embryos were cleared with Hoyer's solution (see following).

\section{XET in vivo co-localisation assay}

The in vivo assay for XET 'action' (=co-localisation of XET activity and the accessible, endogenous donor substrate molecules) was carried out according to Vissenberg et al. (2000) with minor modifications. The xyloglucan-oligosaccharide-sulphorhodamine conjugates (XGO-SRs) were synthesised as described by Fry (1997), with the oligosaccharides consisting of a mixture of XET substrates, mainly the nona-, octa- and hepta-saccharides: XLLG, XXLG and XXXG. These XGO-SRs were dissolved at a concentration of $6.5 \mu \mathrm{M}$ in $25 \mathrm{mM}$ Na-succinate buffer, $\mathrm{pH}$ 5.5; the same concentration of cellobiose-SR was also dissolved in $\mathrm{Na}$ succinate buffer and used as a control.

Cucumber somatic embryos at various stages were collected from the medium and placed for $1 \mathrm{~h}$ in $250 \mu \mathrm{l}$ of cellobiose-SR or XGOs-SR working solutions, in the dark, following the protocol of Vissenberg et al. (2000). For an improved substrate penetration, $1 \mu \mathrm{l}$ of TRITON-X100 (Sigma, St. Louis, Mo.) was added to $250 \mu$ of working solution. The somatic embryos were then incubated in an ethanol:acetic acid (6:1) solution for $1 \mathrm{~h}$, briefly washed with $70 \%$ ethanol and incubated at room temperature for $6 \mathrm{~h}$ in Hoyer's solution (chloral hydrate:glycerol:water, 8:1:2). For subsequent observations, the samples were kept in $10 \%$ glycerol (Sigma) solution. Fluorescence was examined by confocal laser scanning microscopy under an Olympus FV-500 system (Olympus) equipped with a 568-nm argon-krypton laser. The signal was detected between 540 and $520 \mathrm{~nm}$.

\section{Results}

\section{Bioinformatic analysis of the Cs-XTH1 and Cs-XTH3 5' regulatory regions}

Previously, we reported that two cucumber $X T H$ genes, CsXTH1 and CsXTH3, were similarly engaged in somatic embryogenesis (Linkiewicz et al. 2004; Malinowski et al. 2004; Wiśniewska et al. 2012). These genes are also the closest paralogues within 31-member XTH gene family in cucumber and belong to group I/II (Supplementary Figure S1, Supplementary Table S1; Wóycicki et al. 2011). As expected, the nucleotide sequence conservation reaching $75 \%$ identity within coding sequence does not extend to the 5 ' regulatory regions of $\mathrm{Cs} X T H 1$ and $C s X T H 3$ (no significant similarity found). Focusing on the occurrence and distribution of potential regulatory motifs, it is still difficult to find clear similarities in studied regions. Most of the presumptive $c i s$ elements detected were previously characterised as being stress-related (Fig. 1; Chen et al. 2002). Many motifs have been found in the promoters of ethylene- (GCC-box 
and ERE; Ohme-Tagaki et al. 2000), salicylic acid- (W-box, Eulgem et al. 2000, S-box; Kirsch et al. 2000) and ABAinducible genes (W-box, Rushton et al. 1996; ABRE; Busk and Pages 1998; E-box and G-box; Li and Hall 1999). In addition, numerous MYB motifs are usually present in stress-inducible promoters (Chen et al. 2002; Reichmann 2002). E-box elements, which are also present in $C s-X T H$ genes promoters, were found to be the binding site for the BIM1 transcription factor during brassinosteroid signalling (Yin et al. 2005). BR sensing is also an important element of the plant's response to external factors.

\section{Cs-XTH1 and Cs-XTH3 promoter activity in roots}

To determine which promoter region is the most important for the activity of each $C s-X T H$ gene, we used transgenic Arabidopsis plants with promoter deletion fragments fused to a GUS coding sequence. Schematic representation of the constructs used is shown in Fig. 1.

The GUS assay performed on the 7-day-old Arabidopsis seedlings under conditions excluding the involvement of external mechanical stimuli showed that both the Cs-XTHI and $C s-X T H 3$ promoters were active in the roots. This observation is in agreement with previous data from Northern blot analysis where the strongest transcription of both these genes was shown to occur in roots (Malinowski et al. 2004). High promoter activity was observed only in those parts of the root where growth by means of elongation was taking place or had taken place, and no activity was observed in the root meristematic region (Fig. 2). Promoter activity in other parts of the seedlings was low or even undetectable.

Although promoter activity was observed in all of the transformants (Figs. 2, 3a), the shortest promoter fragments $(-275 \mathrm{pCs}-\mathrm{XTH} 1$ and $-244 \mathrm{pCs}-\mathrm{XTH} 3)$ gave only residual GUS staining, whereas longer versions of promoter fragments (i.e. -467 and -928 for $C s-X T H 1,-430$ and -610 for $C s-X T H 3)$ gave comparable staining, mainly in roots of transgenic Arabidopsis seedlings. This suggests that regions from -275 to -467 for $C s$ - XTHI and from -244 to -430 for $C s-X T H 3$ are responsible for the strong expression level.

\section{Influence of mechanical factors}

In a heterologous system (Arabidopsis), both promoters were activated following leaf wounding (Fig. 3a). The shortest promoter fragments (constructs $-275 \mathrm{pCs}-\mathrm{XTH} 1$ and $-244 \mathrm{pCs}-\mathrm{XTH} 3$ ) gave the weakest GUS staining. No differences in promoter fragment activity during the 60-min period between the first and third incision were observed (Fig. 3a, e) in either the homologous (cucumber) or heterologous (Arabidopsis) system. GUS staining of Arabidopsis mature plants showed high promoter activity in tension-bearing regions such as the dehiscence (Fig. 3b, c) and abscission (Fig. 3b) zones of the siliques and flowers (data not shown). Touch-inducible activity of both promoters was also observed (Fig. 3d). Cucumber plants with the $-928 \mathrm{pCs}-\mathrm{XTH} 1$ reporter constructs that were regenerated from somatic embryos also reflected the wound-inducible
Fig. 2 The promoter activity of $C s-X T H 1$ (a) and $C s$-XTH3 (b) genes in Arabidopsis during seedling growth. The images show 7-day-old seedlings grown vertically on the medium surface under vegetative conditions. The highest activity was observed in roots, except for the root tip region. The shortest deletion fragments resulted in only residual GUS activity. The length of the promoter fragments is shown below the images. GUS activity was checked after fixation in $2 \%$ (w/v) paraformaldehyde
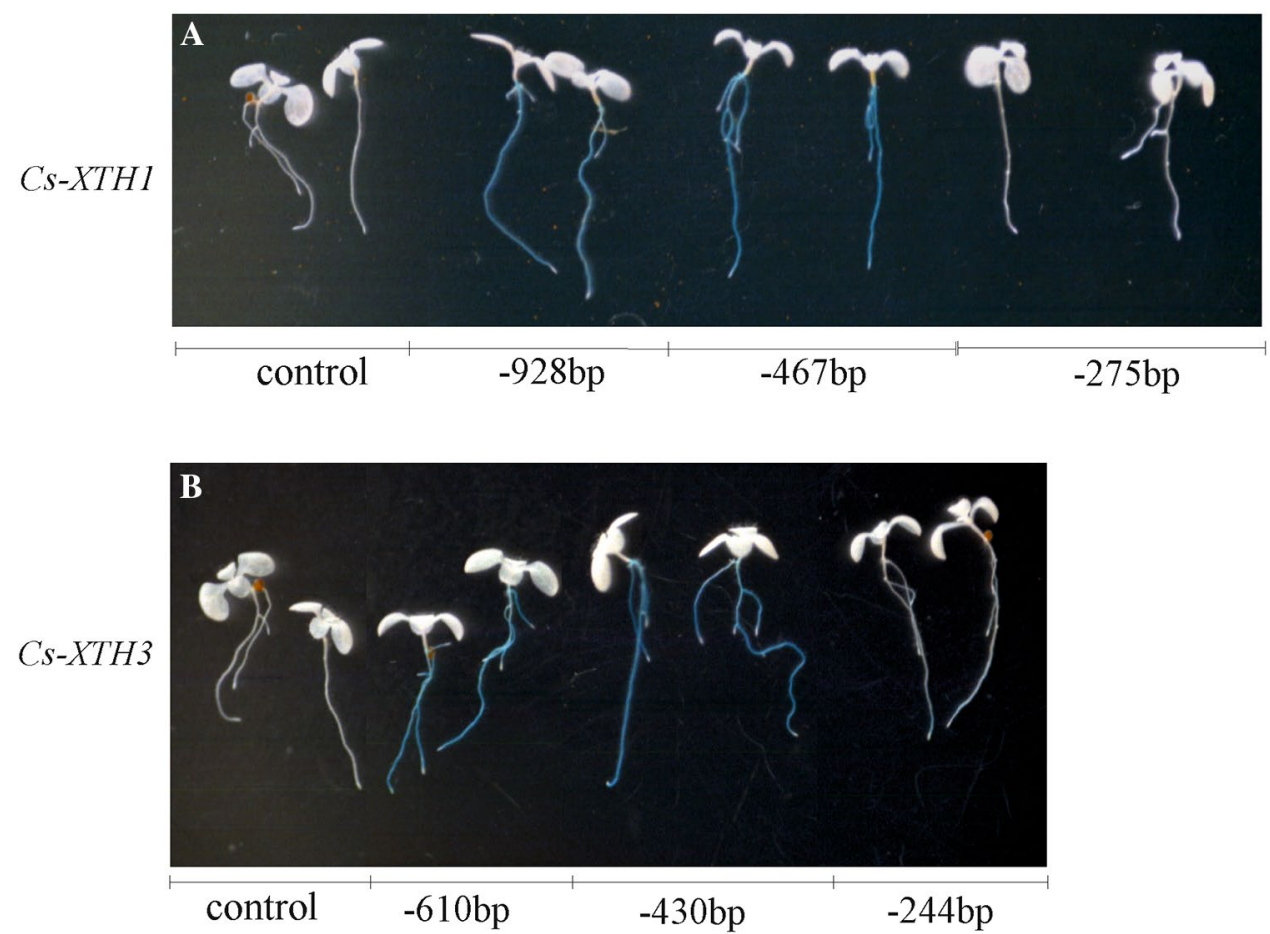
Fig. 3 Mechanical stimuli can induce the $C s-X T H 1$ and $C s-X T H 3$ promoter activity. a Expression patterns in response to wounding by incision. $\mathbf{b}$, c GUS activity revealed by -928pCs-XTHI and -610pCs$X T H 3$ promoter fragments in the tension-bearing regions of the Arabidopsis silique. This pattern was observed for all but the shortest constructs. $\mathbf{d}$ Touch response. For mechanical treatment, Arabidopsis inflorescence stalks were gently touched by the finger tip in sites indicated by arrows. The example shown here is the $-467 \mathrm{pCs}-$ XTH1::GUS transgenic Arabidopsis plant. This response was observed for all but the shortest promoter::GUS fusions. e Wound-inducible activity observed for the -928-bp fragment of the $C s-X T H 1$ promoter in cucumber transgenic plants. The scale bars represent $1 \mathrm{~cm}$
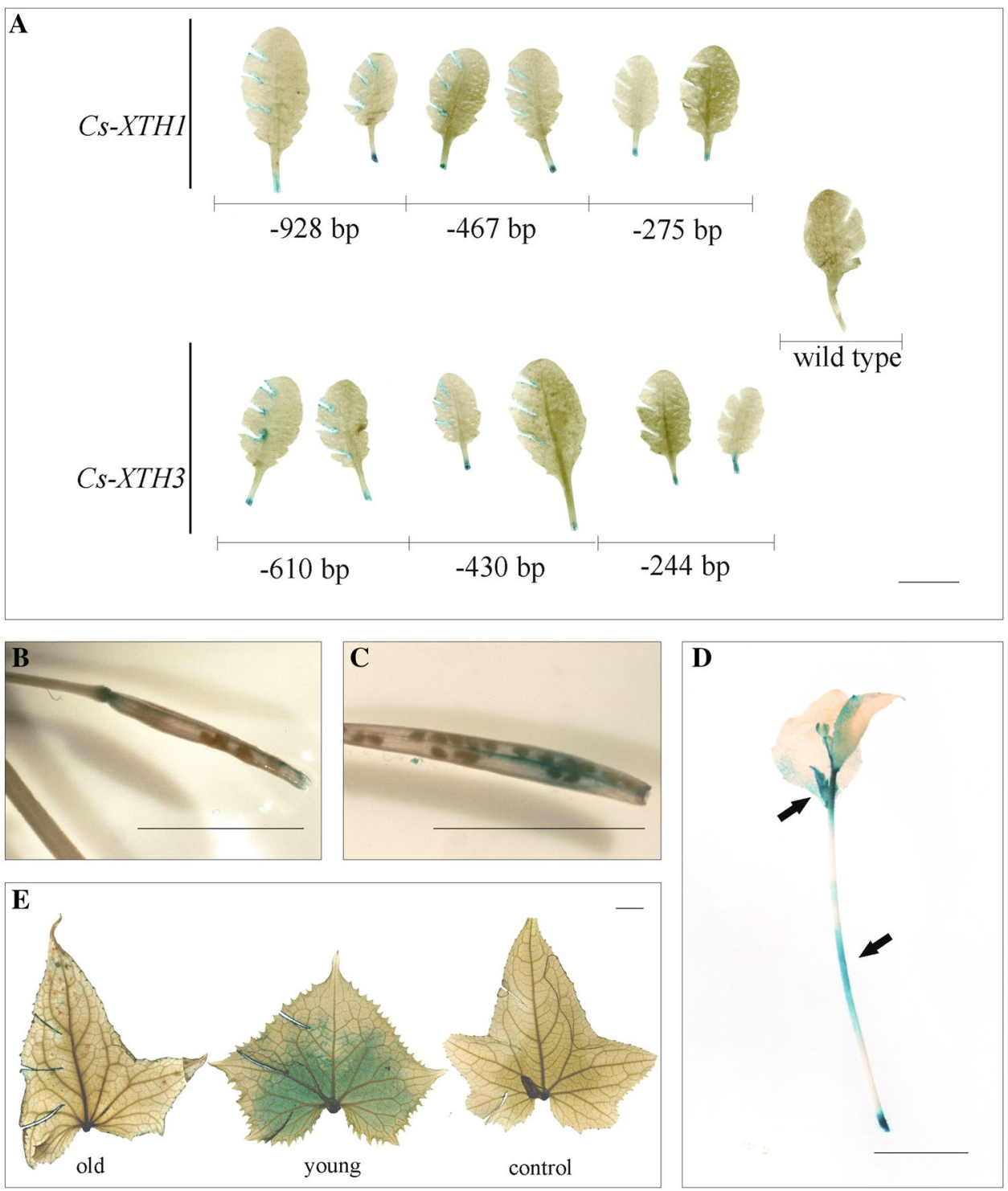

promoter activity; however, additional staining was observed in the central part of younger leaves (Fig. 3e).

\section{The activity of Cs-XTH1 and Cs-XTH3 in cucumber somatic embryos}

Transgenic somatic embryos ( $\mathrm{T}_{0}$ generation) were obtained following the transformation of cucumber embryogenic suspension cultures with the $-928 \mathrm{Cs}-\mathrm{XTH} 1:$ :GUS and -610Cs-XTH3::GUS constructs. The promoter activity observed in transgenic somatic embryos resembled the in situ mRNA localization pattern previously reported for these two genes (Malinowski et al. 2004). The activity of both promoter fragments was strongest in the cotyledonary part of the embryos (Fig. 4c-e, g for $C s-X T H 1$; Fig. $4 \mathrm{~h}-\mathrm{j}$ for $C s-$ $X T H 3)$. In proliferating or degrading somatic embryos, equal
GUS staining of the external cell layers of embryos was observed (Fig. 4f, k) for both promoters. In addition, staining of the differentiated root hairs was observed in germinated somatic embryos (Fig. 4f, k). The strong GUS staining of transgenic cell lines was also observed in small aggregates having the ability for further differentiation. No GUS activity was observed in the control wild-type cucumber somatic embryos (Fig. 4a, b).

\section{In vivo localisation of XET action in cucumber somatic embryos}

Monitoring the gene transcriptional activity (e.g. by promoter::reporter gene fusions) may give a hint in explaining its role. Posttranscriptional, translational, and posttranslational regulations and protein relocation can modify the site of gene action. Moreover in the case of proteins having 
Fig. 4 The activity of the $C s$ $X T H 1$ and $C s-X T H 3$ promoters in cucumber somatic embryos: a, b Control, wild-type somatic embryos; c-g - 928pCs-

XTH1::GUS transgenic somatic embryos; $\mathbf{h}-\mathbf{k}-610$ pCsXTH3::GUS transgenic somatic embryos. Both promoters were most active in the peripheral region of the cotyledons. High promoter activity was also observed in proliferating embryos (f, $\mathbf{k})$ where the blue staining was present in developed root hairs. The scale bars represent $100 \mu \mathrm{m}$
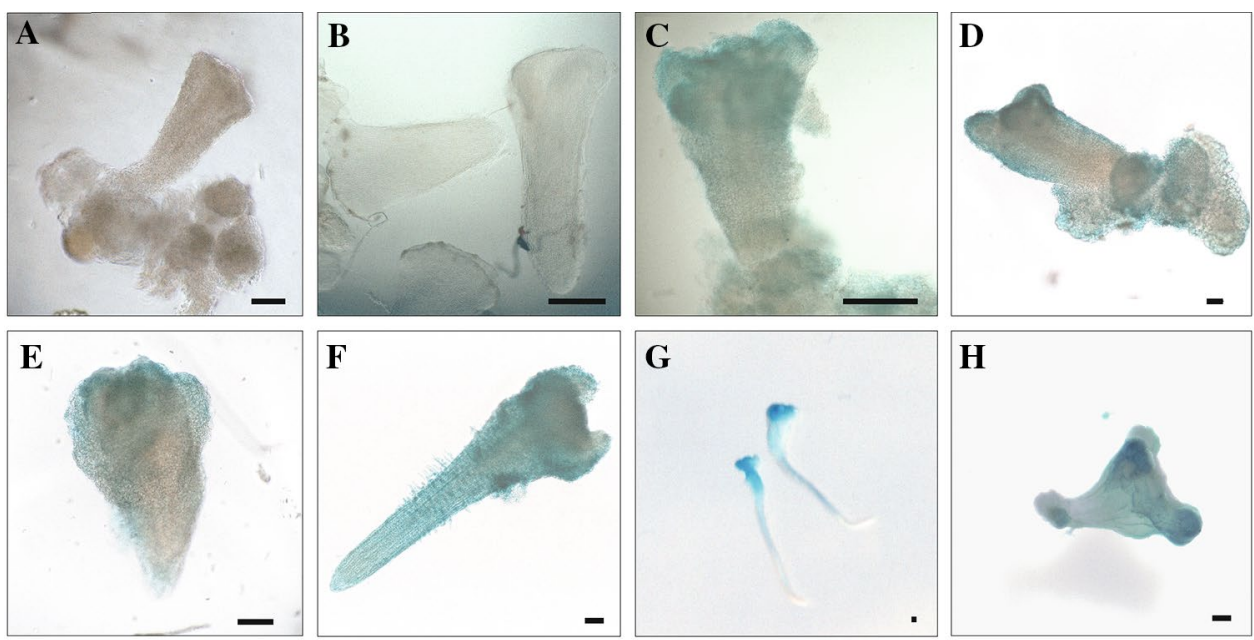

G

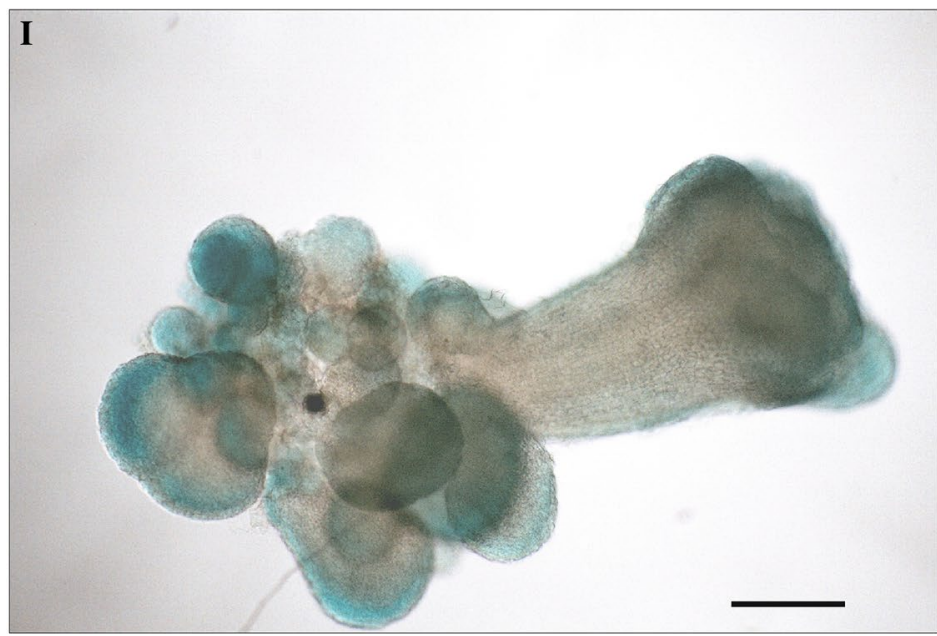

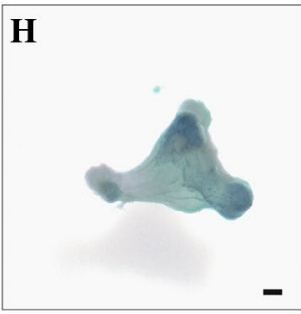
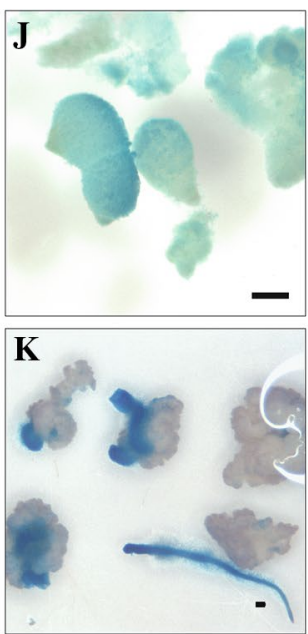

an enzymatic activity, the substrates availability matters. Using the fluorescently labelled acceptor substrate, we may see more precisely where do studied genes act (or other colocalised family members). The localisation of XET action in somatic embryos revealed two different fluorescence patterns corresponding to embryo development modifications observed in embryogenic cucumber cultures. These modifications can be described as shifting the embryo development towards faster growth of apical or basal part. In somatic embryos with arrested or slightly changed development of the cotyledonary parts, the highest XET action was observed in the middle part of the embryo, in an intermediate region between the future root and hypocotyl. This region is known as the presumptive site of peg formation during cucumber seed germination (Takahashi 1997). Observed XET action was specifically localised, and no (or only residual) action was present in the aggregates bearing somatic embryos (Fig. 5a, b). In some in vitro-germinated somatic embryos, strong elongation of this region of high XET action was observed (Fig. 5f). In somatic embryos having well-developed cotyledons, a lower XET action was observed (Fig. 5g, h). However, in this case, the whole embryo body showed fluorescence with the highest XET action being localised in the cotyledons. An extremely strong fluorescence was also observed around the incision of torpedo stage embryo (Fig. 5c). The cell aggregates that accompanied somatic embryos showed strong fluorescence too (Fig. 5i). Only lowlevel fluorescence was observed in control embryos incubated with cellobiose-SR, which is not an effective XET acceptor substrate (this background was also observed for non-incubated embryos): the fluorescence measurements taken after the embryos had been incubated with cellobiose-SR were set as a threshold level for the confocal microscope calibration (Fig. 5j). It should be pointed out the higher-than-background (thus distinguishable) XET action was observed only in the most external layers, except for the root-hypocotyl intermediate region where fluorescence was observed as a ring encompassing several cell layers (Fig. 5e). These data, however, do not exclude the presence of much lower XET activity inside the embryo which was impossible to detect on cellular level in whole-mount experiments because of the relatively big size of the experimental object and, therefore, low magnification used for the confocal microscopy observations. 
Fig. 5 XET action in cucumber somatic embryos. After a 1-h incubation in the cellobioseSR (control-J) or XGOs-SR working solutions, somatic embryos and cell aggregates were observed under the confocal microscope. The pictures in Nomarski optics are shown on the left of each object studied except for $(\mathrm{F})$. The scale bars represent $100 \mu \mathrm{m}$. a XET action in a late heart stage somatic embryo. b XET action in an early torpedo stage somatic embryos. c Torpedo stage wounded. d, e1, e2, f Somatic embryos with arrested cotyledons and strongly elongating root. g Somatic embryo with well-developed cotyledons and elongated root. $\mathbf{h}$ Somatic embryo showing a balanced development, with a proper plumular part. i An aggregate composed of small cells. $\mathbf{j}$ An aggregate with adjacent somatic embryos; control incubation in which the XGO-SR working solution has been replaced by its cellobiose-SR counterpart
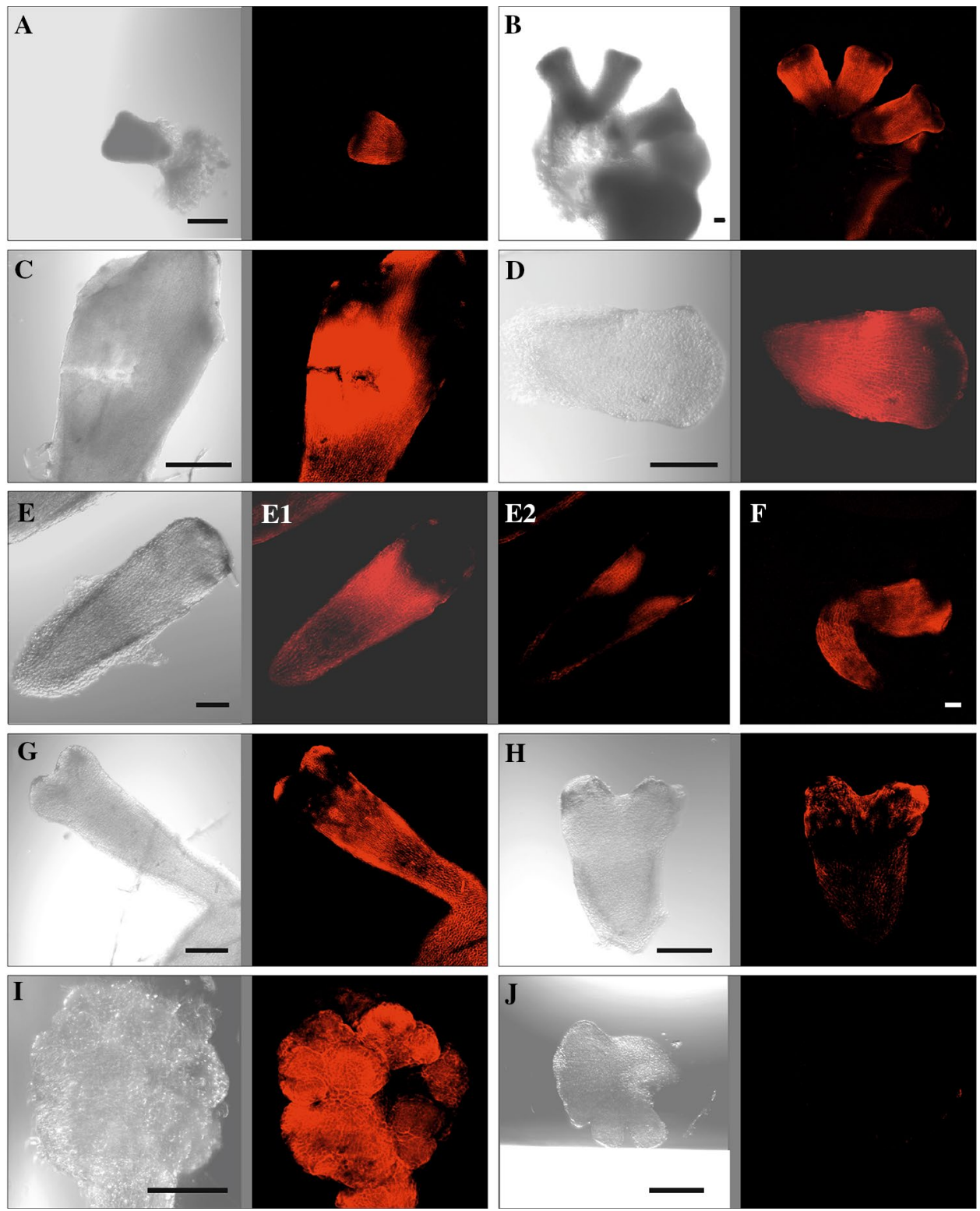

\section{Discussion}

Cucumber embryogenic suspension cultures provide an efficient and useful means of studying gene expression during embryogenesis (Malinowski et al. 2004; Grabowska et al. 2009; Wiśniewska et al. 2012, 2013), mainly because there is no need for plant regeneration and de novo induction of somatic embryogenesis. Previously, we described two cucumber $X T H$ genes, $C s X T H 1$ and $C s X T H 3$, which were involved in somatic embryogenesis (Linkiewicz et al. 2004; Malinowski et al. 2004; Wiśniewska et al. 2012). Here, we present the promoter::GUS fusions analyses of these genes as well as localisation of XET action in cucumber somatic embryos.
The Cs-XTH1 and Cs-XTH3 genes showed developmental regulation. We suggest that this developmental regulation may be correlated to mechanical stress generated internally during tissue growth and differentiation, a proposal which is supported by the activity of both promoters in the silique dehiscence and abscission zones, where several tensions occur (Fig. 3b, c). The strong promoter activity in elongating parts of roots (Fig. 2) and growing cotyledons of cucumber somatic embryos (Fig. 5g, h) can also be related to internal local changes in turgor pressure. The widespread roles of mechanical stimuli in plant development were discussed by Braam (2005).

The observed XET action in regions of the somatic embryos highly responsive to gravity changes (predicted sites of peg formation; Fig. $5 \mathrm{a}, \mathrm{b}, \mathrm{d}-\mathrm{g}$ ) or in cotyledons 
capable of further growth (Fig. $5 \mathrm{~g}$, h) can also be correlated to mechanical changes at the surface of the embryo. When cucumber seeds germinate, they can develop the peg in response to the direction of applied gravity changes (Takahashi et al. 1997). Saito et al. (2004) suggested that this gravistimulation response is related to auxin sensing. During suspension culture growth, the gravitational disturbances are obvious and, consequently, many genes are mechanically activated (Kieran et al. 2000). Soga et al. (2001) reported that xyloglucan metabolism can be modified in response to gravity changes. Such responses could explain the pattern of XET action observed in the cucumber somatic embryos. However, the question of why the plant needs such specific developmental regulation of $X T H$ genes is beyond the scope of the present investigation.

Besides postulated mechanical stress engagement both in plant development and studied genes regulation, there could be other stress factors involved. The diversity of potential development- and stress-related cis elements that we have detected in the regulatory regions of $\mathrm{Cs}-\mathrm{XTH} 1$ and $\mathrm{Cs}-\mathrm{XTH} 3$ (supplementary Table 1) reflects the possible complexity of their regulation. The analysis of GUS activity driven by 5'-truncated promoters of $\mathrm{Cs}$-XTHI and $\mathrm{Cs}$-XTH3 allowed us to identify the promoter regions which are necessary for their efficient transcription. However, further investigations are required to discover the factors which bind to them.

There is a number of stress factors which are often linked to tissue culture and somatic embryogenesis. This in turn can significantly modify embryo development and the activity of particular genes. In contrast to zygotic embryos, which are to some extent isolated from the external environment (seed coat, endosperm, ovule sac), somatic embryos are exposed to external factors that may be able to influence their development. The lack of an endosperm disturbs nutrition, influences the mechanical properties of the region surrounding the embryo and even modifies the size of the embryo (Bonello et al. 2002; Berger 2003; Garcia et al. 2005). A somatic embryo developing in suspension culture may be exposed to hypoxia stress (Merkle et al. 1995). Chapman et al. (2000) reported that one of the most important features during the differentiation and subsequent development of somatic embryos is its ability to arrest or moderate the growth of some cell layers in highly hypotonic conditions (Chapman et al. 2000). Several reports describe the crucial role of cell wall-modifying enzymes, such as glucanases (Helleboid et al. 1998) or peroxidases (Cordewener et al. 1991), in this process.

The promoter activities of $C s-X T H 1$ and $C s-X T H 3$ observed in the heterologous system (Arabidopsis thaliana) used in the present investigation reveal that external mechanical factors are able to play an important role in the regulation of the expression of these genes. Both genes were rapidly induced by wounding (Fig. 3a). Cs-XTH1 promoter activity in the cucumber plants was also induced by wounding (Fig. 3e). As with the XTH22 gene (Braam and Davis 1990), touch induction of both $X T H$ genes was also observed (Fig. 3d).

Based on the results presented here, it would appear that there are two equivalent regulatory mechanisms driving the expression of $\mathrm{Cs}-\mathrm{XTH1}$ and $\mathrm{Cs}-\mathrm{XTH} 3$ - one developmental and the second adaptive. In many of the processes related to embryogenesis, there is a possible interplay between these mechanisms. The expression of many genes active during somatic embryogenesis can be also induced by stress factors (reviewed in Fehér et al. 2003). Moreover, somatic embryogenesis itself can be induced by stress factors (Gaj 2002; Ikeda-Iwai et al. 2003).

We hypothesise that the expression of the two cucumber $X T H$ genes investigated here can be correlated to the need for local cell wall strengthening by the integration of newly secreted xyloglucan molecules during somatic embryo development in a highly stressful environment (Thompson et al. 1997; Sasidharan et al. 2011). However, the involvement of these genes in cell wall loosening by increasing later molecular rearrangement (Thompson and Fry 2001) cannot be also excluded. Moreover, the specific function of the genes analysed should be under additional influence of the activity of redundant $X T H$ family members and molecular structure of substrates available (Eklöf and Brumer 2010; Nishikubo et al. 2011).

Author contribution statement RM carried out the experiments and wrote the manuscript, SCF synthesised XGOSRs, designed experiments with it and worked extensively on the manuscript, SZ and AW did cucumber transformation, MG did confocal microscopy, AN and ABB prepared promoter fusion constructs, SM and MF designed the experiments, analysed data and prepared the final version of the manuscript.

Acknowledgements We thank Dr. Wojciech Burza and Dr. Monika Dobrzyńska for valuable discussions on the embryogenesis. We also thank Dorota Pankowska-Mierzejewska for the excellent technical assistance. Our research was supported by The Foundation for Polish Science and The Polish State Committee for Scientific Research (Grant no 3P06A02325). R. Malinowski and M. Godlewski were funded by a grant from The Foundation for Polish Science Domestic Grant for Young Scientists. Stephen C. Fry thanks the UK BBSRC for funding.

Open Access This article is distributed under the terms of the Creative Commons Attribution 4.0 International License (http://creativeco mmons.org/licenses/by/4.0/), which permits unrestricted use, distribution, and reproduction in any medium, provided you give appropriate credit to the original author(s) and the source, provide a link to the Creative Commons license, and indicate if changes were made. 


\section{References}

Antosiewicz DM, Purugganan MM, Polisensky DH, Braam J (1997) Cellular localization of Arabidopsis xyloglucan endotransglycosylase-related proteins during development and after wind stimulation. Plant Physiol 115:1319-1328

Berger F (2003) Endosperm: the crossroad of seed development. Curr Opin Plant Biol 6:42-50

Bonello J-F, Sevilla-Lecoq S, Berne A, Risueño M-C, Dumas C, Rogowsky PM (2002) Esr proteins are secreted by the cells of the embryo surrounding region. J Exp Bot 53(374):1559-1568

Bourquin V, Nishikubo N, Abe H, Brumer H, Denman S, Eklund M, Christiernin M, Teeri T, Sundberg B, Mellerowicz E (2002) Xyloglucan endotransglycosylases have a function during the formation of secondary dell walls of vascular tissues. Plant Cell 14:3073-3088

Braam J (2005) In touch: plant responses to mechanical stimuli. New Phytol 165:373-389

Braam J, Davis R (1990) Rain-, wind-, and touch-induced expression of calmodulin-related genes in Arabidopsis. Cell 60:357-364

Burza W, Malepszy S (1998) Cytokinin control of cucumber (Cucumis sativus L.) somatic embryogenesis. Book of Abstracts from IX International Congress on Plant Tissue and Cell Culture, Jerusalem, Israel

Burza W, Zuzga S, Yin Z, Malepszy S (2006) Cucumber (Cucumis sativus L.). In: Wang K (ed) Methods in Molecular Biology 343, Agrobacterium protocols. Vol 1. Humana Press Inc., Totowa, pp 427-438

Busk PK, Pages M (1998) Regulation of abscisic acid-induced transcription. Plant Mol Biol 37:425-435

Chanliaud E, De Silva J, Strongitharm B, Jeronimidis G, Gidley MJ (2004) Mechanical effects of plant cell wall enzymes on cellulose/xyloglucan composites. Plant J 38:27-37

Chapman A, Blervacq A-S, Tissier J-P, Delbreil B, Vasseur J, Hilbert J-L (2000) Cell wall differentiation during early somatic embryogenesis in plants. I. Scanning and transmission electron microscopy study on embryos originating from direct, indirect, and adventitious pathways. Can J Bot 78:816-823

Chen W, Provart NJ, Glazebrook J, Katagiri F, Chang H-S, Eulgem T, Mauch F, Luan S, Zou G, Whitham SA, Budworth PR, Tao Y, Xie Z, Chen X, Lam S, Kreps JA, Harper JF, Si-Ammour A, Mauch-Mani B, Heinlein M, Kobayashi K, Hohn T, Dangl JL, Wang X, Zhu T (2002) Expression profile matrix of Arabidopsis transcription factor genes suggests their putative functions in response to environmental stresses. Plant Cell 14:559-574

Clough S, Bent A (1998) Floral dip: a simplified method for Agrobacterium-mediated transformation of Arabidopsis thaliana. Plant J 16:735-743

Cordewener J, Booij H, van der Zandt H, van Engelen F, van Kammen A, De Vries S (1991) Tunicamycin-inhibited carrot somatic embryogenesis can be restored by secreted cationic peroxydase isoenzymes. Planta 184:478-486

De Block M, Debrouwer D (1992) In-situ enzyme histochemistry on plastic-embedded plant material: the development of an artifactfree beta-glucuronidase assay. Plant J 2:261-266

Dumais J, Long SR, Shaw SL (2004) The mechanics of surface expansion anisotropy in Medicago truncatula root hairs. Plant Physiol 136:3266-3275

Eklöf JM, Brumer H (2010) The XTH Gene family: an update on enzyme structure, function, and phylogeny in xyloglucan remodeling. Plant Physiol 153:456-466

Eulgem T, Rushton PJ, Robatzek S, Somssich IE (2000) The WRKY superfamily of plant transcription factors. Trends Plant Sci 5:199-206
Fehér A, Pasternak TP, Dudits D (2003) Transition of somatic plant cells to an embryogenic state. Plant Cell Tissue Organ Cult 74:201-228

Franková L, Fry SC (2013) Biochemistry and physiological roles of enzymes that 'cut and paste' plant cell-wall polysaccharides. J ExpBot 64:3519-3550

Fry SC (1997) Novel 'dot-blot' assays for glycosyltransferases and glycosylhydrolases: optimization for xyloglucan endotransglycosylase (XET) activity. Plant J 11:1141-1150

Gaj MD (2002) Stimulation of somatic embryo formation by mutagens and darkness in culture of immature zygotic embryos of Arabidopsis thaliana (L.) Heynh. Plant Growth Regul 37:93-98

Garcia D, Fitz Gerald JN, Berger F (2005) Maternal control of integument cell elongation and zygotic control of endosperm growth are coordinated to determine seed size in Arabidopsis. Plant Cell 17:52-60

Grabowska A, Wisniewska A, Tagashira N, Malepszy S, Filipecki M (2009) Characterization of CsSEF1 gene encoding putative $\mathrm{CCCH}$-type zinc finger protein expressed during cucumber somatic embryogenesis. J Plant Physiol 166:310-323

Hadi M, Kemper E, Wendeler E, Reiss B (2002) Simple and versatile selection of Arabidopsis transformants. Plant Cell Rep 21:130-135

Hajdukiewicz P, Svab Z, Maliga P (1994) The small versatile pPZP family of Agrobacterium binary vectors for plant transformation. Plant Mol Biol 25:989-994

Hejnowicz Z, Heinemann B, Sievers A (1977) Tip growth: patterns of growth rate and stress in the Chara rhizoid. Z Pflanzenphysiol 81:409-424

Helleboid S, Bauw G, Belingheri L, Vasseur J, Hilbert JL (1998) Extracellular $\beta$-1,3-glucanases are induced during early somatic embryogenesis in Cichorium. Planta 205:56-63

Higo K, Ugawa Y, Iwamoto M, Korenaga T (1999) Plant cis-acting regulatory DNA elements (PLACE) database. Nucleic Acids Res 27:297-300

Ikeda-Iwai M, Umehara M, Satoh S, Kamada H (2003) Stressinduced somatic embryogenesis in vegetative tissues of Arabidopsis thaliana. Plant J 34:107-114

Iliev E, Xu W, Polisensky D, Oh M-H, Torisky R, Clouse SD, Braam J (2002) Transcriptional and posttranscriptional regulation of Arabidopsis TCH4 expression by diverse stimuli. Roles of $c i s$ acting regions and brassinosteroids. Plant Physiol 130:770-783

Kieran P, Malone D, McLoughlin P (2000) Effects of hydrodynamic and interfacial forces on plant cell suspension systems. Adv Biochem Eng Biot 67:141-177

Kirsch C, Takamiya-Wik M, Schmelzer E, Hahlbrock K, Somssich IE (2000) A novel regulatory element involved in rapid activation of parsley ELI7 gene family members by fungal elicitor or pathogen infection. Mol Plant Pathol 1:243-251

Li G, Hall TC (1999) Footprinting in vivo reveals changing profiles of multiple factor interactions with the $\beta$-phaseolin promoter during embryogenesis. Plant J 18:633-641

Linkiewicz A, Filipecki M, Tomczak A, Grabowska A, Malepszy S (2004) The cloning of sequences differentially transcribed during the induction of somatic embryogenesis in cucumber (Cucumis sativus L.). Cell Mol Biol Lett 9:795-804

Malinowski R, Filipecki M, Tagashira N, Wiśniewska A, Gaj P, Plader W, Malepszy S (2004) Xyloglucan endotransglucosylase/hydrolase genes in cucumber (Cucumis sativus) - differential expression during somatic embryogenesis. Physiol Plant 120:678-685

Maris A, Suslov D, Fry SC, Verbelen J-P, Vissenberg K (2009) Enzymic characterization of two recombinant xyloglucan endotransglucosylase/hydrolase (XTH) proteins of Arabidopsis and their effect on root growth and cell wall extension. J Exp Bot 60:3959-3972 
Merkle SA, Parrott WA, Flinn BS (1995) Morphogenic aspects of somatic embryogenesis. In: Thorpe TA (ed) In vitro embryogenesis in plants. Kluwer, Dordecht, pp 155-203

Murashige T, Skoog T (1962) A revised medium for rapid growth and bioassays with tobacco tissue culture. Physiol Plant 15:473-497

Nakamura T, Yokoyama R, Tomita E, Nishitani K (2003) Two Azuki Bean XTH genes, VaXTH1 and VaXTH2, with similar tissue-specific expression profiles, are differently regulated by auxin. Plant Cell Physiol 44:16-24

Nishikubo N, Takahashi J, Roos AA, Derba-Maceluch M, Piens K, Brumer H, Teeri TT, Stålbrand H, Mellerowicz EJ (2011) Xyloglucan endo-transglycosylase-mediated xyloglucan rearrangements in developing wood of hybrid aspen. Plant Physiol 155:399-413

Nishitani K (2002) A genome-based approach to study the mechanisms by which cell-wall type is defined and constructed by the collaborative actions of cell-wall-related enzymes. J Plant Res 115:303-307

Ohme-Tagaki M, Suzuki K, Shinshi H (2000) Regulation of ethylene-induced transcription of defense genes. Plant Cell Physiol 41:1187-1192

Potters G, Pasternak TP, Guisez Y, Palme KJ, Jansen MAK (2007) Stress-induced morphogenic responses: growing out of trouble? Trends Plant Sci 12:98-105

Potters G, Pasternak TP, Guisez Y, Jansen MAK (2009) Different stresses, similar morphogenic responses: integrating a plethora of pathways. Plant Cell Environ 32:158-169

Riechmann JL (2002) Transcriptional regulation: a genomic overview. In: Somerville CR, Meyerowitz EM (eds) The Arabidopsis book. American Society of Plant Biologists, Rockville, pp 1-46

Rushton PJ, Torres JT, Parnicke M, Wernert P, Hahlbrock K, Somssich IE (1996) Interaction of elicitor-induced DNA binding proteins with elicitor response elements in the promoters of parsley PR1 genes. EMBO J 15:5690-5700

Saito Y, Yamasaki S, Fujii N, Hagen G, Guilfoyle T, Takahashi H (2004) Isolation of cucumber CsARF cDNAs and expression of the corresponding mRNAs during gravity-regulated morphogenesis of cucumber seedlings. J Exp Bot 55:1315-1323

Sasidharan R, Voesenek LA, Pierik R (2011) Cell wall modifying proteins mediate plant acclimatization to biotic and abiotic stresses. Crit Rev Plant Sci 30:548-562

Shahmuradov IA, Gammerman AJ, Hancock JM, Bramley PM, Solovyev VV (2003) PlantProm: a database of plant promoter sequences. Nucleic Acids Res 31:114-117

Sharples SC, Nguyen-Phan CT, Fry SC (2017) Xyloglucan endotransglucosylase/hydrolases (XTHs) are inactivated by binding to glass and cellulosic surfaces, and released in active form by a heat-stable polymer from cauliflower florets. J Plant Physiol 218:135-143

Soga K, Wakabayashi K, Hoson T, Kamisaka S (2001) Gravitational force regulates elongation growth of Arabidopsis hypocotyls by modifying xyloglucan metabolism. Adv Space Res 27:1011-1016

Takahashi H (1997) Gravimorphogenesis: gravity-regulated formation of the peg in cucumber seedlings. Planta 203:S164-S169

Thompson JE, Fry SC (2001) Restructuring of wall-bound xyloglucan by transglycosylation in living plant cells. Plant J 26:23-34

Thompson JE, Smith RC, Fry SC (1997) Xyloglucan undergoes interpolymeric transglycosylation during binding to the plant cell wall in vivo: evidence from ${ }^{13} \mathrm{C} /{ }^{3} \mathrm{H}$ dual labelling and isopycnic centrifugation in caesium trifluoroacetate. Biochem J 327:699-708
Vissenberg K, Martinez-Vilchez IM, Verbelen JP, Miller JG, Fry SC (2000) In vivo co-localisation of xyloglucan endotransglycosylase activity and its donor substrate in the elongation zone of Arabidopsis roots. Plant Cell 12:1229-1237

Vissenberg K, Fry SC, Verbelen J-P (2001) Root hair initiation is coupled to a highly localized increase of xyloglucan endotransglycosylase action in Arabidopsis roots. Plant Physiol 127:1125-1135

Vissenberg K, Van Sandt V, Fry SC, Verbelen J-P (2003) Xyloglucan endotransglucosylase action is high in the root elongation zone and in the trichoblasts of all vascular plants from Selaginella to Zea mays. J Exp Bot 54:335-344

Vissenberg K, Oyama M, Osato Y, Yokoyama R, Verbelen J-P, Nishitani K (2005) Differential expression of AtXTH17, AtXTH18, AtXTH19 and AtXTH20 genes in Arabidopsis roots. Physiological roles in specification in cell wall construction. Plant Cell Physiol 46:192-200

Wiśniewska A, Grabowska A, Pietraszewska-Bogiel A, Tagashira N, Zuzga S, Wóycicki R, Przybecki Z, Malepszy S, Filipecki M (2012) Identification of genes up-regulated during somatic embryogenesis of cucumber. Plant Physiol Biochem 50:54-64

Wiśniewska A, Pietraszewska-Bogiel A, Zuzga S, Tagashira N, Łotocka B, Malepszy S, Filipecki M (2013) Molecular analysis of SCARECROW (CsSCR) gene expressed during somatic embryo development and in root of cucumber (Cucumis sativus L.). Acta Physiol Plantarum 35:1483-1495

Wóycicki R, Witkowicz J, Gawroński P, Dąbrowska J, Lomsadze A et al (2011) The genome sequence of the north-european cucumber (Cucumis sativus L.) unravels evolutionary adaptation mechanisms in plants. PLoS One 6:e22728

Xu W, Purugganan M, Polisensky D, Antosiewicz D, Fry SC, Braam J (1995) Arabidopsis TCH4, regulated by hormones and the environment, encodes a xyloglucan endotransglycosylase. Plant Cell 7:1555-1567

Xu W, Campbell P, Vargheese A, Braam J (1996) The Arabidopsis XET-related gene family: environmental and hormonal regulation of expression. Plant J 9:879-889

Ye X, Yuan S, Guo H, Chen F, Tuskan GA, Cheng Z-M (2012) Evolution and divergence in the coding and promoter regions of the Populus gene family encoding xyloglucan endotransglycosylase/ hydrolases. Tree Genet Genomes 8:177-194

Yin Y, Vafeados D, Tao Y, Yoshida S, Asami T, Chory J (2005) A new class of transcription factors mediates brassinosteroid-regulated gene expression in Arabidopsis. Cell 120:249-259

Yokoyama R, Nishitani K (2001) A comprehensive expression analysis of all members of a gene family encoding cell-wall enzymes allowed us to predict $c i s$-regulatory regions involved in cell-wall construction in specific organs of Arabidopsis. Plant Cell Physiol 42:1025-1033

Yokoyama R, Rose JKC, Nishitani K (2004) A surprising diversity and abundance of xyloglucan endotransglucosylase/hydrolases in rice. Classification and expression analysis. Plant Physiol 134:1088-1099

Yokoyama R, Uwagaki Y, Sasaki H, Harada T, Hiwatashi Y, Hasebe M, Nishitani K (2010) Biological implications of the occurrence of 32 members of the XTH (xyloglucan endotransglucosylase/ hydrolase) family of proteins in the bryophyte Physcomitrella patens. Plant J 64:645-656 